\title{
Crystalline plasticity in isotactic polypropylene below and above the glass transition temperature
}

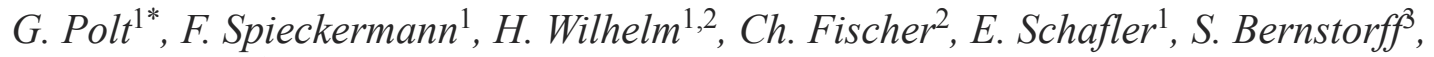 \\ M. Zehetbauer ${ }^{1}$ \\ ${ }^{1}$ Research Group Physics of Nanostructured Materials, Faculty of Physics, University of Vienna, Boltzmanngasse 5, \\ Vienna, Austria \\ ${ }^{2}$ Laboratory of Polymer Engineering LKT-TGM, Wexstrasse 19-23, 1200 Vienna, Austria \\ ${ }^{3}$ Sincrotrone Trieste, Strada Statale 14 km 163.5 in AREA Science Park, 34149 Basovizza, Trieste, Italy
}

Received 9 March 2015; accepted in revised form 15 May 2015

\begin{abstract}
In-situ X-ray diffraction was applied to isotactic polypropylene with a high volume fraction of $\alpha$-phase ( $\alpha$-iPP) while it has been compressed at temperatures below and above its glass transition temperature $T_{\mathrm{g}}$. The diffraction patterns were evaluated by the Multi-reflection X-ray Profile Analysis (MXPA) method, revealing microstructural parameters such as the density of dislocations and the size of coherently scattering domains (CSD-size). A significant difference in the development of the dislocation density was found compared to compression at temperatures above $T_{\mathrm{g}}$, pointing at a different plastic deformation mechanism at these temperatures. Based on the individual evolutions of the dislocation density and CSD-size observed as a function of compressive strain, suggestions for the deformation mechanisms occurring below and above $T_{\mathrm{g}}$ are made.
\end{abstract}

Keywords: thermal properties, polypropylene, dislocation, X-ray diffraction, plasticity

\section{Introduction}

Semicrystalline polymers consist of a complex multiscale structure. A physical interpretation of deformation processes at the macroscopic scale can only be successful when both, micro- and mesoscopic deformation processes are considered. At the mesoscopic scale, rearrangements of lamellar crystalline blocks such as bending, rotation, fragmentation or shear band formation occur [1]. At the microscopic scale, basic micromechanisms of deformation within the crystalline and amorphous phase are active.

There is already an overwhelming amount of data demonstrating that the plasticity of semicrystalline polymers is controlled by the deformation of the crystalline phase even up to large strains [2]. In this connection, two deformation mechanisms have been discussed, those are a) an approach based on adiabatic melting and recrystallization [3], and b) the deformation by crystallographic slip [4]. The major part of publications, favor the occurrence of the latter mechanism even for a wide range of strain [5-7]. Recently it could be shown that this mechanism is governed by nucleation and propagation of dislocations [8-10], although it was also reported [9] that in some polymer systems other deformation modes have to be considered in the crystalline phase. Nevertheless, the amorphous phase still contributes to the deformation process as the stresses are being transmitted between the two phases.

In the present work, the deformation behaviour of the crystalline phase - with special respect to a dislocation mediated mechanism - has been investigated at

\footnotetext{
${ }^{*}$ Corresponding author, e-mail: gerald.polt@univie.ac.at

(C) BME-PT
} 
different deformation temperatures, in order to find out the role of the amorphous phase having varying rigidity. Therefore, in-situ compression experiments during X-ray diffraction below the glass transition temperature $\left(T_{\mathrm{g}}\right)$ were performed on polypropylene with a high volume fraction of $\alpha$-phase and compared with previous observations done above $T_{\mathrm{g}}$.

\section{Experimental}

\subsection{Material and measurement}

Isotactic polypropylene (type BE50 from Borealis) was used during this investigation (melt flow rate $\left(230^{\circ} \mathrm{C} / 2,16 \mathrm{~kg}\right): 0,30 \mathrm{~g} / 10 \mathrm{~min}$, density: $\left.905 \mathrm{~kg} / \mathrm{m}^{3}\right)$. Cylindrical samples were produced with $6 \mathrm{~mm}$ in diameter and a height of $10 \mathrm{~mm}$.

In-situ Wide Angle X-ray Scattering (WAXS) measurements during compression below the glass transition temperature were performed at the Synchrotron Light Source ELETTRA in Trieste, Italy (SAXSBeamline 5.2L). Measurements were performed with a photon energy of $8 \mathrm{keV}$ which corresponds to a wavelength of $\lambda=1.54 \AA$, close to $\mathrm{CuK} \alpha$ radiation. The spot size on the sample was $500 \mu \mathrm{m} \times 150 \mu \mathrm{m}$ while measurements were performed in a transmission setup which ensured a large sample volume to be investigated. Diffraction patterns were recorded using a curved position sensitive detector of the type INEL CPS-590. In order to reach high degrees of deformation without cavitation, the sample was deformed by compression using a miniature compression machine mounted to the sample stage. After a WAXS pattern was recorded of the undeformed sample at a temperature of $24.8^{\circ} \mathrm{C}$, the sample was cooled below its glass transition temperature of 0 , to $-5^{\circ} \mathrm{C}$ using an Oxford Cryostream Cooler. The sample was then deformed stepwise, still being at a temperature of $-5^{\circ} \mathrm{C}$, using a true strain rate of $\dot{e}=10^{-3} \mathrm{~s}^{-1}$ to different levels of strain up to a true plastic strain of $e=0.8$. The deformation was stopped every time a X-ray diffraction pattern was recorded.

\subsection{Multi-reflection $X$-ray profile analysis (MXPA)}

The diffraction patterns were evaluated using the Multi-reflection X-ray profile analysis (MXPA) method which allows for a detailed analysis of diffraction profiles revealing parameters such as the coherently scattering domain size (CSD-size, also called 'crystal size' in literature) and the density of dislocations [11-15]. A physical line profile of a Bragg reflection can be expressed by the convolution of the intensity profiles originating from the limited crystal size and by lattice strains, such as from dislocations. In Fourier space the contributions of size and strain can be well separated resulting in the Warren-Averbach equation (Equation (1)) [16]:

$\ln A_{\mathrm{L}}=\ln A_{\mathrm{L}}^{\mathrm{S}}+\ln A_{\mathrm{L}}^{\mathrm{D}}=\ln A_{\mathrm{L}}^{\mathrm{S}}+\exp \left(-2 \pi^{2} L^{2} g^{2}\left\langle\varepsilon_{\mathrm{g}, \mathrm{L}}^{2}\right)\right.$

where $A_{\mathrm{L}}^{\mathrm{S}}$ and $A_{\mathrm{L}}^{\mathrm{D}}$ are the size and the distortion coefficients respectively, with the Fourier Length $L$. The distortion coefficient depends on the absolute value of the diffraction vector $K=2 \sin \theta / \lambda$, where $K=g$ at the exact Bragg-position and the mean square strain $\left\langle\varepsilon_{\mathrm{g}, \mathrm{L}}^{2}\right\rangle$. The distortion coefficient is modeled under the assumption that the mean square strain originates from the strain field of dislocations [17], as shown by Equation (2):

$$
\left\langle\varepsilon_{\mathrm{g}, \mathrm{L}}^{2}\right\rangle=\frac{\rho \bar{C} b^{2}}{4 \pi} \ln \left(\frac{R_{\mathrm{e}}}{L}\right)
$$

with the absolute value of the Burgers vector $b$, the dislocation density $\rho$ and the effective outer cut-off radius of dislocations $R_{\mathrm{e}}$. The peak broadening due to dislocations is taken into account by the average dislocation contrast factor $\bar{C}[17,18]$, while the size coefficient is calculated assuming a lognormal size distribution of the crystals [12].

\subsection{Evaluation of MXPA}

Before the evaluation procedure, the background scattering and the $\alpha$ and $\gamma$-phase have been modeled by analytical functions using the program fityk [19] (Figure 1). Only the $\alpha$-phase was used for the final evaluation using the programs $C M W P-f i t$ [12] and its extension Multi-Eval [20], since it could be shown that dislocations play only a minor role during the plastic deformation in the $\gamma$-phase [21]. Details of the evaluation procedure have already been described in a previous work [21], however it is noticed that the error bars in Figure 2 and 3 do not represent a physical error, but a numerical one indicating the reproducibility of the evaluation process. 


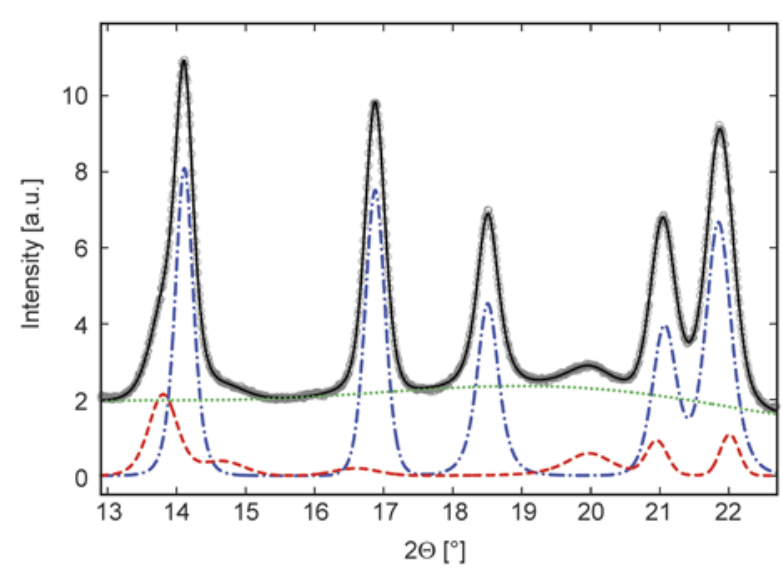

Figure 1. WAXS pattern of undeformed iPP below $T_{\mathrm{g}}$. Measured data (o), fit (full line), $\alpha$-phase (dashdotted line), $\gamma$-phase (dashed line) and amorphous phase (dotted line).

\section{Results}

At the beginning of in-situ compression below the glass transition temperature $T_{\mathrm{g}}$, a yield stress of $120 \mathrm{MPa}$ was observed in the true stress - true strain plot. This value is higher than the flow stress occurring during compression at room temperature mostly because of the increased stiffness of the amorphous phase.

The development of the dislocation density as a function of true strain is shown in Figure 2. In case of the room temperature experiment, the dislocation density increases markedly at a plastic strain of approximately 0.2 from initially $\rho=0.5 \cdot 10^{15}$ to $\rho=$ $1.6 \cdot 10^{16} \mathrm{~m}^{-2}$. In contrast to that, the change in dislocation density of the sample deformed below $T_{\mathrm{g}}$ is

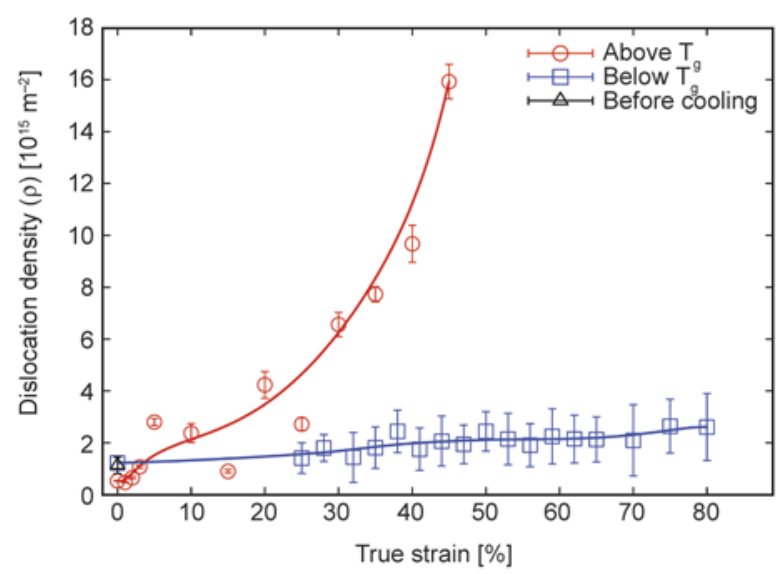

Figure 2. Development of the dislocation density of $\alpha$-iPP at room temperature and below its glass transition. The black triangle indicates the dislocation density obtained for the unloaded sample at a temperature of $24.8^{\circ} \mathrm{C}$ before cooling below $T_{\mathrm{g}}$.

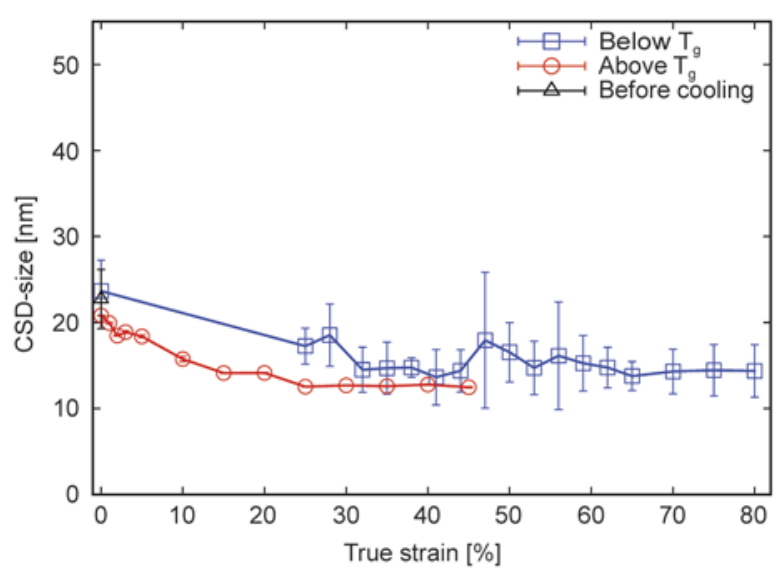

Figure 3. The coherently scattering domain size (CSD-size) as a function of true strain. The black triangle indicates the CSD-size obtained for the unloaded sample at a temperature of $24.8^{\circ} \mathrm{C}$ before cooling below $T_{\mathrm{g}}$. Some refinement of the undefected areas is found.

moderate: The dislocation density of initially $\rho=$ $1 \cdot 10^{15} \mathrm{~m}^{-2}$ increases slightly up to $\rho=2.6 \cdot 10^{15} \mathrm{~m}^{-2}$. In Figure 3, the development of the coherently scattering domain size (CSD-size) is depicted as a function of deformation. The CSD-size represents the smallest domain which scatters coherently. Assuming a cuboid like structure of the crystalline domains in the lamellae $[10,21,22]$, the CSD-size can be related to the thickness of the lamellae. A decrease in the CSD-size can be seen at both deformation temperatures, from 20 to $12 \mathrm{~nm}$ for the sample deformed beyond $T_{\mathrm{g}}$ and from 23 to $14 \mathrm{~nm}$ for the deformation below $T_{\mathrm{g}}$.

Crystallinity was found to decrease linearly from initially $66 \%$ to a value of $52 \%$ at a plastic deformation of $e=0.8$ for the sample deformed below $T_{\mathrm{g}}$ and from 56 to $43 \%$ for the room temperature experiment as evaluated from X-ray diffractograms.

\section{Discussion}

Much experimental data are available in literature which confirm that deformation of polymer crystals operates via crystallographic slip $[5,6,8,23,24]$. In the case of $\alpha$-iPP deformed at room temperature i.e. above the glass transition temperature $T_{\mathrm{g}}$, crystal slip is facilitated by dislocation motion since an increasing number of dislocations are nucleated as deformation proceeds (Figure 2). The situation is markedly different when $\alpha$-iPP is compressed at temperatures below its glass transition $T_{\mathrm{g}}$ where only a weak increase in dislocation density is observed pointing at another deformation mechanism being active. 
Not at least due to the presence of tie molecules which connect the crystalline lamellar phase with the amorphous one, deformation occurs in each of the phases. Especially at deformations above $T_{\mathrm{g}}$, the soft viscoelastic behavior of the amorphous phase provides additional degrees of freedom to the lamellar crystals. Here, for the deformation of the amorphous phase, three deformation modes can occur which are (i) interlamellar shear (ii) interlamellar separation and (iii) lamellae stack rotation [25-28]. During mechanism (i) the amorphous region between two crystals is exposed to shear with the shear direction being parallel to the lamellae. Interlamellar separation (ii) is characterized by a change in the spacing between two lamellae, and lamellae stack rotation (iii) causes a whole set of parallel lamellae to rotate as a unit.

In contrast, the plastic deformation of the crystalline phase in polypropylene can be the result of crystallographic slip processes [26], or twinning [29-31]. However, the slip process can accommodate also large amounts of deformation, and is thus quoted in most works to carry most of the plastic strain [26]. Crystallographic slip occurs first on the closest packed planes in the unit cell. During slip, two parts of a polymer crystal, separated by the slip plane undergo a relative translation against each other, which is achieved (at least in the actual case of $\alpha$-iPP) by dislocation motion along the slip plane. However, dislocations only move if the force on the dislocation and as a consequence, the resolved shear stress $\tau$ reaches a critical value $\tau_{0}$ (critical resolved shear stress) that is characteristic for the corresponding slip system.

The resolved shear stress for uniaxial tension or compression by a stress $\sigma$ is given by $\tau=$ $\sigma \cdot \cos \kappa \cdot \cos \varphi=m \sigma$ where $\kappa$ and $\varphi$ are the angles of the slip plane normal and the slip direction with respect to the uniaxial stress $\sigma$, respectively, and $m$ is the Schmid-factor which can vary within 0 and 0.5 . It is assumed that the preferred slip type especially at smaller deformations is chain slip, operating along the chain direction [32]. Slip transverse to the chain direction is known to be energetically less favored due to its higher $\tau_{0}[33,34]$.

At early stages of deformations above $T_{\mathrm{g}}$, deformation primarily occurs in the amorphous phase by one or more of the possible deformation mechanisms discussed above, while the hard crystalline lamellae with ongoing deformation start to rotate within the soft amorphous matrix [28]. When the slip planes are tilted with respect to the direction of the applied stress $\sigma$, the Schmid-factor changes accordingly leading to a change in the resolved shear stress. If $\tau_{0}$ is reached due to chain orientation, more polymer crystals will experience crystallographic slip, accompanied by dislocation motion along the slip plane. With proceeding deformation, additional dislocations are generated to maintain the plastic flow, leading to an increase in the density of dislocations, especially exceeding a true strain of about 25\% (Figure 2).

Below the glass transition temperature, however, the deformation of the amorphous phase, and thus, the rotation of polymer crystals are hindered. As a result, the lamellae are prohibited in rotating to a position where the Schmid-factor reaches a maximum, therefore less crystals undergo plastic slip which - in the actual material - means that markedly less dislocations are generated compared to the same material deformed at room temperature, above $T_{\mathrm{g}}$. In such a purely crystallographic picture we can conclude that the amorphous phase is acting similarly to an additional slip system as for instance suggested by Nikolov and Raabe [35]. On a molecular scale, however, the nucleation of new dislocations is also related to molecular relaxations in the crystalline phase. Some authors have suggested thermally activated chain twist defects to be responsible for the nucleation of dislocations [36]. It is possible that the reduction of conformational degrees of freedom below $T_{\mathrm{g}}$ of polypropylene is also inhibiting the nucleation of such precursor defects for dislocations [36]. In this context a manuscript is in preparation studying the annihilation of dislocations in polypropylene as a function of molecular relaxation processes [Polt G, unpublished].

As a consequence of the starvation of dislocations for deformations below $T_{\mathrm{g}}$, one can - independently of the underlying mechanism - expect that the deformation is accompanied by micro-cracking and or shear banding, and that the lamellae are deformed by fragmentation before crystallographic slip occurs. Such behavior was already observed in poly(3hydroxybutyrate) (P3HB) where no increase in the dislocation density at larger strains could be found due to considerable crack formation and shear banding [9].

However, a reduction in CSD-size is seen in both experiments, below and above $T_{\mathrm{g}}$ (Figure 3 ). This 
reduction is the result of any deformation that breaks the coherence in the crystal. If the misorientation exceeds an angle of $1.5^{\circ}$ within the crystal, these two parts of the crystal will be registered as two separate domains by the MXPA method. Below $T_{\mathrm{g}}$ the primary deformation mechanism operating in the crystalline phase is bending of the lamellae involving the formation of geometrically necessary misfit dislocations [21], accompanied by only small amounts of crystallographic slip due to the hindered rotation of the lamellae, consequently leading to a continuous decrease in the domain size involving an only moderate increase in the dislocation density; the situation seems to be very similar to the plastic deformation of $\gamma$-modification of Polypropylene [21].

In case of the room temperature experiment (above $T_{\mathrm{g}}$ ), it can be assumed that both mechanisms, i.e. bending of the lamellae including misfit dislocations, and crystallographic slip are operating simultaneously over the whole strain range. However, the distinct reduction in the CSD-size up to a true strain of $25 \%$ can primarily be attributed to misfit dislocations, operating simultaneously with crystallographic slip processes on slip planes with a low $\tau_{0}$; namely chain slip planes with moving screw dislocations [26, 34].

Exceeding a true strain of about $25 \%$, deformation by bending of the lamellae and therefore the decrease in the CSD-size becomes less dominant, while additional slip systems are being activated due to the higher resolved shear stress. The activation of additional transverse slip dislocations with edge character stimulates a pronounced increase of the dislocation density, in addition to the increasing number of screw dislocations in the chain slip system [34].

The decrease in crystallinity by $14 \%$ is most probably caused by the amorphization of the crystalline domains due to shear band formation and/or by lamellar fragmentation through micro-cracking.

\section{Conclusions}

$\alpha$-phase polypropylene was plastically deformed below and above its glass transition temperature and has been observed in-situ by X-ray line profile analysis using synchrotron radiation.

The resulting microstructural parameters such as the size of the coherently scattering domains and the density of dislocations were evaluated as a func- tion of deformation up to true strains of $e=0.8$. Significant differences in the development of the dislocation density were observed which can be explained as follows:

1) The distinct increase in the dislocation density above the glass transition temperature originates from crystallographic slip processes which are accompanied by dislocation generation and motion.

2) The less pronounced increase in dislocation density below the glass transition temperature results from the reduced ability of the polymer crystals to move and rotate in the amorphous matrix due to its increased stiffness in this temperature range. Therefore less slip systems reach the critical resolved shear stress, being necessary to initiate slip and/or the lamellae even undergo fragmentation without showing any crystallographic slip. However small amounts of dislocations are being created by geometrically necessary misfit dislocations accompanied by only small fractions of crystallographic slip. Crystalline deformation may operate via shear band formation also being accompanied by micro-cracking.

3) The decrease in the CSD-size observed in the present experiments at deformations below and above $T_{\mathrm{g}}$ most probably arises from bending of the lamellae leading to a misorientation within the crystal under the formation of geometrically necessary dislocations. However, exceeding a true strain of about $25 \%$ above $T_{\mathrm{g}}$, the main deformation mechanism being active within the crystals changes to crystallographic slip. As a consequence, the CSD-size remains constant while a significant increase of the dislocation density is observed.

\section{References}

[1] Bartczak Z., Galeski A.: Plasticity of semicrystalline polymers. Macromolecular Symposia, 294, 67-90 (2010).

DOI: $10.1002 /$ masy.201050807

[2] Oleinik E. F.: Plasticity of semicrystalline flexiblechain polymers at the microscopic and mesoscopic levels. Polymer Science Series C, 45, 17-117 (2003).

[3] Flory P. J., Yoon D. Y.: Molecular morphology in semicrystalline polymers. Nature, 272, 226-229 (1978). DOI: 10.1038/272226a0

[4] Young R.: A dislocation model for yield in polyethylene. A dislocation model for yield in polyethylene. Philosophical Magazine, 30, 85-94 (1974).

DOI: $\underline{10.1080 / 14786439808206535}$ 
[5] Bartczak Z., Argon A. S., Cohen R. E.: Deformation mechanisms and plastic resistance in single-crystal-textured high-density polyethylene. Macromolecules, 25, 5036-5053 (1992).

DOI: $10.1021 / \mathrm{ma} 00045 \mathrm{a} 034$

[6] Gleiter H., Argon A. S.: Plastic deformation of polyethylene crystals. Philosophical Magazine, 24, 71-80 (1971).

DOI: $10.1080 / 14786437108216425$

[7] Séguéla R.: Plasticity of semi-crystalline polymers: Crystal slip versus melting-recrystallization. E-polymers, 7, 382-401 (2007).

DOI: 10.1515/epoly.2007.7.1.382

[8] Wilhelm H., Paris A., Schafler E., Bernstorff S., Bonarski J., Ungar T., Zehetbauer M. J.: Evidence of dislocations in melt-crystallised and plastically deformed polypropylene. Materials Science and Engineering: A, 387-389, 1018-1022 (2004).

DOI: $10.1016 /$ j.msea.2004.03.099

[9] Spieckermann F., Polt G., Wilhelm H., Kerber M., Schafler E., Zehetbauer M. J.: The role of dislocations for the plastic deformation of semicrystalline polymers as investigated by multireflection X-ray line profile analysis. Journal of Applied Polymer Science, 125, 4150-4154 (2012).

DOI: $10.1002 / a p p .36570$

[10] Spieckermann F., Wilhelm H., Kerber M., Schafler E., Polt G., Bernstorff S., Addiego F., Zehetbauer M.: Determination of lamella thickness distributions in isotactic polypropylene by X-ray line profile analysis. Polymer, 51, 4195-4199 (2010).

DOI: 10.1016/j.polymer.2010.07.009

[11] Ribárik G.: Modelling of diffraction patterns based on microstructural properties. $\mathrm{PhD}$ Thesis, Eötvös Loránd University (2008).

[12] Ribárik G., Ungár T., Gubicza J.: MWP-fit: A program for multiple whole-profile fitting of diffraction peak profiles by $a b$ initio theoretical functions. Journal of Applied Crystallography, 34, 669-676 (2001). DOI: $10.1107 /$ S0021889801011451

[13] Ungár T., Borbély A.: The effect of dislocation contrast on X-ray line broadening: A new approach to line profile analysis. Applied Physics Letters, 69, 31733175 (1996).

DOI: $10.1063 / 1.117951$

[14] Kerber M. B., Zehetbauer M. J., Schafler E., Spieckermann F., Bernstorff S., Ungar T.: X-ray Line profile analysis - An ideal tool to quantify structural parameters of nanomaterials. Journal of the Minerals, Metals and Materials Society, 63, 61-70 (2011).

DOI: $10.1007 / \mathrm{s} 11837-011-0115-1$

[15] Schafler E., Zehetbauer M.: Characterization of nanostructured materials by X-ray line profile analysis. Reviews on Advanced Materials Science, 10, 28-33 (2005).
[16] Warren B. E., Averbach B. L.: The effect of cold-work distortion on X-ray patterns. Journal of Applied Physics, 21, 595-599 (1950).

DOI: $10.1063 / 1.1699713$

[17] Wilkens M.: The determination of density and distribution of dislocations in deformed single crystals from broadened X-ray diffraction profiles. Physica Status Solidi (a), 2, 359-370 (1970).

DOI: 10.1002/pssa.19700020224

[18] Krivoglaz M.: Theory of X-ray and thermal neutron scattering by real crystals. Plenum Press, New York (1969).

[19] Wojdyr M.: Fityk: A general-purpose peak fitting program. Journal of Applied Crystallography, 43, 11261128 (2010). DOI: $10.1107 / \mathrm{S} 0021889810030499$

[20] Kerber M.: X-ray line profile analysis in theory and experiment. PhD Thesis, University of Vienna (2012).

[21] Polt G., Spieckermann F., Wilhelm H., Kerber M., Schafler E., Bernstorff S., Zehetbauer M.: The role of dislocations in $\gamma$-iPP under plastic deformation investigated by X-ray line profile analysis. Mechanics of Materials, 67, 126-132 (2013).

DOI: $10.1016 /$ j.mechmat.2013.05.010

[22] Baltá-Calleja F., Cruz C., Bayer R., Kilian H.: Microhardness and surface free energy in linear polyethylene: The role of entanglements. Colloid and Polymer Science, 268, 440-446 (1990).

DOI: $10.1007 / \mathrm{BF} 01411002$

[23] Seguela R., Staniek E., Escaig B., Fillon B.: Plastic deformation of polypropylene in relation to crystalline structure. Journal of Applied Polymer Science, 71, 1873-1885 (1999).

DOI: $10.1002 /($ SICI) 1097-4628(19990314)71:11<1873 :AID-APP18>3.0.CO;2-I

[24] Bartczak Z., Galeski A.: Yield and plastic resistance of $\alpha$-crystals of isotactic polypropylene. Polymer, 40, 3677-3684 (1999). DOI: $10.1016 / \mathrm{S} 0032-3861(98) 00614-4$

[25] Phillips A., Zhu P-W., Edward G.: Simple shear deformation of polypropylene via the equal channel angular extrusion process. Macromolecules, 39, 5796-5803 (2006).

DOI: $10.1021 / \mathrm{ma} 0607618$

[26] Galeski A.: Strength and toughness of crystalline polymer systems. Progress in Polymer Science, 28, 16431699 (2003).

DOI: $10.1016 / \mathrm{j}$. progpolymsci.2003.09.003

[27] Lin L., Argon A. S.: Structure and plastic deformation of polyethylene. Journal of Materials Science, 29, 294-323 (1994). DOI: $10.1007 / \mathrm{BF} 01162485$

[28] Hirsch J. R., Wang P. T.: Texture and strength evolution in deformed polypropylene. Textures and Microstructures, 13, 101-122 (1994).

DOI: $10.1155 /$ TSM.13.101 
[29] Kojima M.: Morphology of polypropylene crystals. II. Twinning of lamellar crystals. Journal of Polymer Science Part A 2: Polymer Physics, 5, 615-622 (1967). DOI: $10.1002 /$ pol.1967.160050317

[30] Hibi S., Niwa T., Mizukami J., Wang C., Kyu T.: Crystal orientation and twinning in cold-rolled ultrahigh molecular weight polypropylene. Polymer Engineering and Science, 35, 911-920 (1995). DOI: 10.1002/pen.760351105

[31] Bassett D. C., Olley R. H.: On the lamellar morphology of isotactic polypropylene spherulites. Polymer, 25, 935-943 (1984).

DOI: 10.1016/0032-3861(84)90076-4

[32] Jia J., Raabe D.: Evolution of crystallinity and of crystallographic orientation in isotactic polypropylene during rolling and heat treatment. European Polymer Journal, 42, 1755-1766 (2006).

DOI: $\underline{10.1016 / \text { j.eurpolymj.2006.02.013 }}$
[33] Bartczak Z., Martuscelli E.: Orientation and properties of sequentially drawn films of an isotactic polypropylene/ hydrogenated oligocyclopentadiene blend. Polymer, 38, 4139-4149 (1997). DOI: $10.1016 / \mathrm{S} 0032-3861(96) 00996-2$

[34] Spieckermann F.: Investigation of deformation induced changes of the microstructure of semicrystalline polymers and their impact on mechanical properties. $\mathrm{PhD}$ Thesis, University of Vienna (2010).

[35] Nikolov S., Raabe D.: Yielding of polyethylene through propagation of chain twist defects: Temperature, stem length and strain-rate dependence. Polymer, 47, 16961703 (2006).

DOI: $10.1016 /$ j.polymer.2005.12.050

[36] Boyd R. H.: Relaxation processes in crystalline polymers: Molecular interpretation - A review. Polymer, 26, 1123-1133 (1985).

DOI: $10.1016 / 0032-3861(85) 90240-\mathrm{X}$ 